\title{
An adaptive optimized Runge-Kutta-Nyström method for second-order IVPs
}

\author{
Mufutau Ajani Rufai ${ }^{1}$, Francesca Mazzia ${ }^{1}$, and HIGINIO RAMOS ${ }^{2}$ \\ ${ }^{1}$ University of Bari Aldo Moro Department of Informatics \\ ${ }^{2}$ University of Salamanca
}

February 20, 2022

\begin{abstract}
This research paper is concerned with developing, analyzing, and implementing an adaptive optimized one-step block Nyström method for solving second-order initial value problems of ODEs and time-dependent partial differential equations. The new technique is developed through a collocation method with a new approach for selecting the collocation points. An embeddinglike procedure is used to estimate the error of the proposed optimized method. The current approach has produced approximate solutions to real-world oscillatory, periodic and stiff application problems. The numerical experiments demonstrate that the introduced error estimation and stepsize control strategy presented in this manuscript has produced a good performance compared with some of the other existing numerical methods.
\end{abstract}

\section{Hosted file}

MFHmanuscript .pdf available at https ://authorea . com/users/461478/articles/557169-an-adaptiveoptimized-runge-kutta-nystr\%C3\%B6m-method-for-second-order-ivps 